\title{
Competing effects of interactions and spin-orbit coupling in a quantum wire
}

\author{
V. Gritsev ${ }^{1}$ G. I. Japaridze, ${ }^{2}$ M. Pletyukhov ${ }^{3}$ and D. Baeriswyl ${ }^{1}$ \\ ${ }^{1}$ Département de Physique, Université de Fribourg, CH-1700 Fribourg, Switzerland \\ ${ }^{2}$ Institute of Physics, Georgian Academy of Sciences, Tamarashvili 6, 0177 Tbilisi, Georgia \\ ${ }^{3}$ Institut für Theoretische Festkörperphysik, Universität Karlsruhe, D-76128 Karlsruhe, Germany
}

\begin{abstract}
We study the interplay of electron-electron interactions and Rashba spin-orbit coupling in onedimensional ballistic wires. Using the renormalization group approach we construct the phase diagram in terms of Rashba coupling, Tomonaga-Luttinger stiffness and backward scattering strength. We identify the parameter regimes with a dynamically generated spin gap and show where the Luttinger liquid prevails. We also discuss the consequences for the operation of the Datta-Das transistor.
\end{abstract}

PACS numbers: 71.70.Ej

The main goal of recent studies in the field of spintronics is to invent ways of manipulating the electron spin with an efficiency comparable to that of presentday electronics (which manipulates charge) 1]. One of the elementary spintronic devices, the Datta-Das transistor [2], has been proposed more than a decade ago, its basic ingredient being a ballistic quantum wire with sufficiently strong Rashba spin-orbit interaction [3]. The latter is required for creating a sizeable spin precession. Depending on spin orientations in the source and in the drain one can modulate the current flowing through the device and thus implement in principle ON/OFF states. The strength of the spin-orbit coupling can be tuned by applying a gate voltage to the system [4].

To understand the feasibility of such a device as well as its basic operation it is important to investigate the effects of spin-orbit coupling on both transport and magnetic properties of (essentially one-dimensional) interacting electrons. Recently some progress towards a solution of this problem has been made on the basis of the Tomonaga-Luttinger model [5]. In the present article we show that the a priori assumption of validity of this approximation is not always justified. We find in fact that the combined effects of Coulomb interaction and Rashba coupling can generate a spin gap and thus radically change the physical characteristics of the DattaDas transistor. In particular, we establish the parameter range where the correlation functions decay exponentially, and the Datta-Das device becomes non-operating.

We consider a narrow ballistic wire described by a onedimensional model of interacting electrons, including the Rashba-type spin-orbit coupling, $V_{R}=\alpha_{R} \sigma_{y} p_{x}$, where $\alpha_{R}$ is the coupling strength and $\sigma_{y}$ is a Pauli matrix.

The electron-electron interaction (assumed to be weak) may be decomposed into the different scattering processes [6]. We include not only the standard forward scatterings of the Tomonaga-Luttinger model (coupling parameters $\left.g_{1 \|}, g_{2 \|}, g_{2 \perp}, g_{4 \|}, g_{4 \perp}\right)$, but also the backward scattering term $\left(g_{1 \perp}\right)$. At the same time we do neglect Umklapp processes $\left(g_{3}\right)$ since the system considered here is far away from half-filling.
Although in the absence of spin-orbit coupling (and for repulsive electron-electron interactions) the backscattering term is usually irrelevant, this is not always the case in the presence of spin-orbit coupling. As will be shown below, the combined effects of these two processes together with the forward scattering lead to the dynamical generation of a spin gap in the excitation spectrum in a wide range of coupling constants. In this range the ground state phase diagram qualitatively differs from that of the Tomonaga-Luttinger model used before [5, 7].

We assume weak bare couplings and therefore linearize the single-particle spectrum around the two Fermi points $\pm k_{F}$. Decomposing the field operators in the standard way [6] into right $(r=+)$ and left movers $(r=-), \psi_{\sigma}(x)=\sum_{r} \psi_{r, \sigma}(x) \exp \left(i r k_{F} x\right)$, we obtain the continuum limit of the fermionic Hamiltonian $H=$ $H_{0}+H_{R}+H_{\text {int }}$, where $H_{0}$ and $H_{\text {int }}$ are the usual oneand two-particle parts, respectively, while the spin-orbit term reads

$H_{R}=i \alpha_{R} k_{F} \sum_{r= \pm} r \int d x\left(\psi_{r, \uparrow}^{\dagger}(x) \psi_{r, \downarrow}(x)-\psi_{r, \downarrow}^{\dagger}(x) \psi_{r, \uparrow}(x)\right)$.

We remark that terms involving gradients of the fields $\psi_{r, \sigma}(x)$ have been neglected because they would generate irrelevant operators for non-vanishing electronelectron interactions 8]. All parts of the Hamiltonian can be bosonized in terms of fields $\phi_{c}(x), \theta_{c}(x)$ for the charge and $\phi_{s}(x), \theta_{s}(x)$ for the spin degrees of freedom, with commutation relations $\left[\phi_{\lambda}(x), \theta_{\lambda^{\prime}}\left(x^{\prime}\right)\right]=$ $(-i / 2) \delta_{\lambda \lambda^{\prime}} \operatorname{sign}\left(x-x^{\prime}\right)$ [] $]$. The Rashba term

$$
H_{R}=\frac{4 \alpha_{R} k_{F}}{\pi \alpha} \int d x \sin \left(\sqrt{2 \pi} \phi_{s}\right) \sin \left(\sqrt{2 \pi} \theta_{s}\right)
$$

(with a short distance cut-off $\alpha$ ) involves only $\phi_{s}$ and $\theta_{s}$ and therefore does not affect spin-charge separation (the same holds for the backscattering term). The kinetic energy and the forward scattering terms become $H=H_{c, 0}+H_{s, 0}$, where the charge part $H_{c, 0}$ and the free spin part $H_{s, 0}$ both have the familiar TomonagaLuttinger form,

$$
H_{\lambda, 0}=\frac{u_{\lambda}}{2} \int d x\left[K_{\lambda}\left(\partial_{x} \theta_{\lambda}\right)^{2}+\frac{1}{K_{\lambda}}\left(\partial_{x} \phi_{\lambda}\right)^{2}\right] .
$$


The parameters $u_{\lambda}, K_{\lambda}, \lambda=c, s$ depend in a simple way on the coupling constants $g_{1, \|}, g_{2, \|}, g_{2, \perp}, g_{4, \|}, g_{4, \perp}$ [6, 9].

Treating $H_{R}$ as a perturbation we notice that its vacuum expectation value vanishes (a consequence of nonzero conformal spin). Thus the lowest-order effect of the Rashba one-particle process is seen to be absent, even though the process is strongly relevant. One then has to study the higher-order expansions in order to find contributions with zero conformal spin (cf. Ref. [9, 10]). The second-order contribution (proportional to $\alpha_{R}^{2}$ ) corresponds to effective two-particle interactions

$$
\psi_{+, \uparrow}^{\dagger} \psi_{-, \uparrow} \psi_{-, \downarrow}^{\dagger} \psi_{+, \downarrow}+\psi_{+, \uparrow}^{\dagger} \psi_{+, \downarrow} \psi_{-, \uparrow}^{\dagger} \psi_{-, \downarrow}+\text { h.c. },
$$

a first term describing a backscattering process already included in the Hamiltonian and a second term representing a spin-nonconserving process which has not been met before. We therefore have to add the new interaction term in the renormalization procedure, with a vanishing initial coupling constant. In this way the operator product expansion governing the renormalization group (RG) flow is closed. We also notice that the process in question usually emerges in the field-theoretical description of systems with completely broken spin-rotational symmetry [11, 14].

The bosonized Hamiltonian for the spin part can be written as $H_{s}=H_{s, 0}+H_{R}+H_{\mathrm{bf}}$, where $H_{s, 0}$ and $H_{R}$ have been given before and $H_{\mathrm{bf}}$ includes the backscattering and spin flip parts, cf. Eq. (4),

$H_{\mathrm{bf}}=\frac{2 g_{1 \perp}}{(2 \pi \alpha)^{2}} \int d x \cos \left(\sqrt{8 \pi} \phi_{s}\right)+\frac{2 g_{f}}{(2 \pi \alpha)^{2}} \int d x \cos \left(\sqrt{8 \pi} \theta_{s}\right)$.

The Hamiltonian $H_{s}$ has the dual symmetry

$$
\phi_{s} \longleftrightarrow \theta_{s}, \quad K_{s} \longleftrightarrow K_{s}^{-1}, \quad g_{1 \perp} \longleftrightarrow g_{f},
$$

which will be used below to obtain the phase diagram of the model in the full parameter range. Thus, if a transition occurs for some set of parameters there must be also a transition for the dual set of parameters.

Standard perturbative RG analysis [12] up to the third order in couplings yields the set of equations

$$
\begin{aligned}
\frac{d y_{R}}{d l} & =\left(4-\left(K_{s}+K_{s}^{-1}\right)\right) y_{R}+y_{\perp} y_{f} y_{R}, \\
\frac{d y_{\perp}}{d l} & =2\left(1-K_{s}\right) y_{\perp}+\left(K_{s}^{-1}-K_{s}\right) y_{R}+\frac{y_{\perp}}{4}\left(y_{\perp}^{2}-y_{f}^{2}\right), \\
\frac{d y_{f}}{d l} & =2\left(1-K_{s}^{-1}\right) y_{f}-\left(K_{s}^{-1}-K_{s}\right) y_{R}-\frac{y_{f}}{4}\left(y_{\perp}^{2}-y_{f}^{2}\right), \\
\frac{d K_{s}}{d l} & =\frac{1}{2}\left(y_{f}^{2}-y_{\perp}^{2} K_{s}^{2}\right),
\end{aligned}
$$

where $l$ measures the logarithm of the length scale, $y_{\perp}=g_{1 \perp} / \pi u_{s}, y_{f}=g_{f} / \pi u_{s}$ and $y_{R}=\left(2 \alpha_{R} k_{F} \alpha / \pi u_{s}\right)^{2}$. Note that it is $y_{R}$ that enters (7), not $\alpha_{R}$. The RG equations are therefore independent of $\operatorname{sign}\left(\alpha_{R}\right)$, a consequence of time-reversal symmetry. The initial conditions are $K_{s}(0) \equiv K_{0}, y_{\perp}(0) \equiv y_{\perp, 0}, y_{R}(0) \equiv y_{R, 0}$ and $y_{f}(0)=0$. Similar RG equations have been derived in the context of spinless fermions on a ladder 9,10 . The RG equations (7) have three weak-coupling fixed points: (I) $y_{\perp}^{*}=y_{f}^{*}=y_{R}^{*}=0$ and $K_{s}^{*}$ arbitrary ; (IIa,b) $K_{s}^{*}=1$, $y_{R}^{*}=0, y_{f}^{*}= \pm y_{\perp}^{*}$ and $y_{\perp}^{*}$ arbitrary. The spin-wave fixed point 13] (I) corresponds to the noninteracting system with no spin-orbit coupling. The fixed points (IIa,b) correspond to the critical Ashkin-Teller (AT) model [13, 14].

A rough idea of the RG flow can be given by assuming the spin stiffness $K_{s}$ to be constant $\left(=K_{0}\right)$ and by neglecting cubic terms in (7). The solutions are

$$
\begin{aligned}
y_{R}(l) & =y_{R, 0} e^{l \gamma_{R}}, \\
y_{\perp,(f)}(l) & =A_{\perp,(f)} e^{2 l\left[1-K_{0}^{(-1)}\right]}+B_{\perp,(f)} e^{l \gamma_{R}},
\end{aligned}
$$

where $B_{\perp,(f)}= \pm y_{R, 0}\left[K_{0}^{-1}-K_{0}\right] /\left[2 \pm K_{0} \mp K_{0}^{-1}\right]$ and $A_{\perp,(f)}=y_{\perp,(f), 0}-B_{\perp,(f)}$. The one-particle Rashba process is relevant if the exponent $\gamma_{R}=\left[4-\left(K_{0}+K_{0}^{-1}\right)\right]$ is positive, i.e. for $2-\sqrt{3}<K_{0}<2+\sqrt{3}$. The amplitude $y_{R, 0}$ is larger than both $B_{\perp}$ and $B_{f}$ for $(\sqrt{5}-1) / 2<K_{0}<(\sqrt{5}+1) / 2$. This (Rashba dominated) region will be investigated more accurately below. For $K_{0} \leq(\sqrt{5}-1) / 2$, backscattering $\left(y_{\perp}\right)$ dominates, while for $K_{0} \geq(\sqrt{5}+1) / 2$, spin flip processes $\left(y_{f}\right)$ prevail, in agreement with the duality relations (66).

We return now to the full RG equations (7). Numerical solutions for various initial conditions in the regime of dominant spin-orbit coupling are illustrated in Fig. 1 as flows in the $\left(y_{R}, \frac{1}{2} \ln K_{s}\right)$ plane. The results confirm that $K_{s}$ remains essentially constant for $y_{\perp, 0}=0$ and is weakly renormalized for $y_{\perp, 0}=0.4$. On the other hand, the Rashba coupling $y_{R}$ rapidly grows from its initial value $y_{R, 0}$ up to some value of order 1 at a length $l_{R}$, while the couplings $y_{\perp}$ and $y_{f}$ remain small. It is then possible

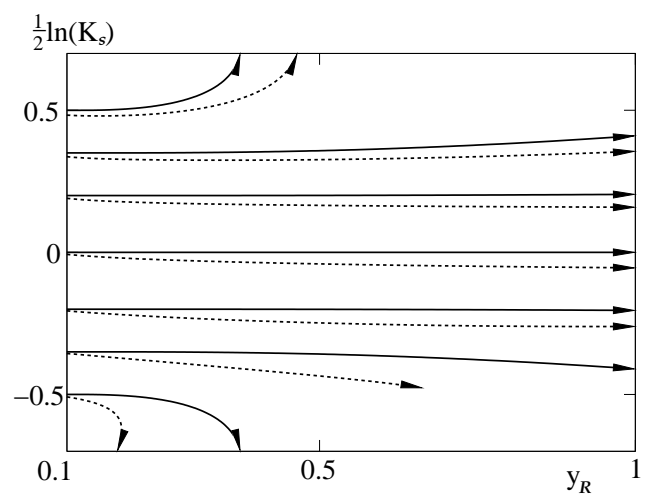

FIG. 1: Flow diagram for the $\left(y_{R}, \frac{1}{2} \ln K_{s}\right)$ plane for $y_{R, 0}=$ 0.07 and various initial values $K_{0}$. Full lines correspond to $y_{\perp, 0}=0$, dashed lines to $y_{\perp, 0}=0.4$.

to add a second step in the RG procedure [9] by treating $H_{R}$ at $l_{R}$ non-perturbatively through the canonical transformation $\psi_{r, \pm}=\frac{1}{\sqrt{2}}\left(\psi_{r, \uparrow \downarrow}-i \psi_{r, \downarrow \uparrow}\right)$. The trans- 
formed single-particle Hamiltonian is

$$
H_{0}+H_{R}=\sum_{r, r^{\prime}= \pm} \int d x \psi_{r, r^{\prime}}^{\dagger}(x)\left(r v_{F} \partial_{x}+r^{\prime} \alpha_{R} k_{F}\right) \psi_{r, r^{\prime}}(x)
$$

Its spectrum consists of two subbands split horizontally by $2 \alpha_{R} k_{F} / v_{F}$, where $v_{F}=u_{s} K_{s}$ is the renormalized Fermi velocity. The canonical transformation leads to the same type of interaction terms as in the original Hamiltonian, with coupling constants $\tilde{y}_{\perp, f} \approx$ $K_{s}-1+\left(y_{\perp, f}-y_{f, \perp}\right) / 2, \tilde{K}_{s}-1 \approx\left(y_{\perp}+y_{f}\right) / 2$ (for $\left|K_{s}-1\right| \ll 1$; otherwise the relations are slightly more complicated).

Bosonization can be applied to the new Hamiltonian in terms of fields $\tilde{\phi}_{s}$ and $\tilde{\theta}_{s}$. The Rashba term leads to a contribution $(\sqrt{2} / \pi) \alpha_{R} k_{F} \int d x \partial_{x} \tilde{\theta}_{s}$ which can be absorbed in the single-particle part by the shift

$$
\Theta_{s}(x)=\tilde{\theta}_{s}(x)+k_{R} x, \quad \Phi_{s}(x)=\tilde{\phi}_{s}(x),
$$

where $k_{R}=\sqrt{2} \alpha_{R} k_{F} /\left(\pi u_{s} \tilde{K}_{s}\right)$. The spin part of the new Hamiltonian reads

$$
\begin{aligned}
\tilde{H}_{s}= & \frac{u_{s}}{2} \int d x\left\{\tilde{K}_{s}\left(\partial_{x} \Theta_{s}\right)^{2}+\frac{1}{\tilde{K}_{s}}\left(\partial_{x} \Phi_{s}\right)^{2}\right. \\
& \left.+\frac{\tilde{y}_{\perp}}{\pi \alpha^{2}} \cos \left(\sqrt{8 \pi} \Phi_{s}\right)-\frac{\tilde{y}_{f}}{\pi \alpha^{2}} \cos \left(\sqrt{8 \pi} \Theta_{s}-k_{R} x\right)\right\} .
\end{aligned}
$$

For $\tilde{K}_{s}>1$ the ordering in the $\Theta_{s}$ sector and the Rashba process are competing, but since for $y_{R} \approx 1$ and $\tilde{y}_{f} \ll$ 1 the last term is wiped out by strong oscillations, the subsequent flow involves only the parameters $\tilde{K}_{s}$ and $\tilde{y}_{\perp}$. The equations of the second RG step have the form of the Kosterlitz-Thouless flow

$$
\frac{d \tilde{K}_{s}}{d l}=-\frac{1}{2} \tilde{y}_{\perp}^{2}, \quad \frac{d \tilde{y}_{\perp}}{d l}=2\left(1-\tilde{K}_{s}\right) \tilde{y}_{\perp},
$$

with the initial conditions $\tilde{K}_{s}(0)=1+\frac{1}{2}\left(y_{\perp}\left(l_{R}\right)+y_{f}\left(l_{R}\right)\right)$, $\tilde{y}_{\perp}(0)=K_{s}\left(l_{R}\right)-1+\frac{1}{2}\left(y_{\perp}\left(l_{R}\right)-y_{f}\left(l_{R}\right)\right)$. Eqs. (11) imply two distinct scenarios, for $\left|\tilde{y}_{\perp}\right|>2\left[\tilde{K}_{s}(0)-1\right]$ a flow to strong coupling and for $\left|\tilde{y}_{\perp}\right|<2\left[\tilde{K}_{s}(0)-1\right]$ a flow to the weak-coupling fixed line $\tilde{y}_{\perp}^{*}=0, \tilde{K}_{s}^{*}$ (non-universal renormalized value). Clearly the line $y_{\perp}=y_{f}, K_{s}=1$ is critical for arbitrary $y_{R}$. We find systematically the weak-coupling case (for $y_{\perp, 0} \geq 0$ ). Thus, if the flow is dominated by the Rashba term, the system is a Luttinger liquid, with enhanced spin precession.

We discuss now the parameter regions where the Rashba term is not dominant, and the RG flow is governed by Eqs. (7). We find numerically that for $K_{s} \neq 1$ either $y_{\perp}$ or $y_{f}$ are renormalized towards strong coupling, $y_{\perp}$ for $K_{s}<1, y_{f}$ for $K_{s}>1$, in agreement with the approximate analytical solutions (8). Both cases scale to the strong-coupling regime of the sine-Gordon model and therefore must have a spin gap $\Delta_{s}$ in the excitation spectrum. At the same time, the Rashba term and the band splitting are dynamically suppressed. This is an unexpected new result, because in the absence of the bare spin-orbit coupling there is no spin gap for $y_{\perp} \geq 0$.

Our findings can be interpreted in terms of two commensurate-incommensurate transitions [15]. In the incommensurate phase the Rashba term dominates and the effective field theory is equivalent to a TomonagaLuttinger model with non-universal spin stiffness and momentum shift (9). In the commensurate phases the spin excitations have a gap, produced by backscattering and spin flip processes, respectively. Fig. 2 shows the

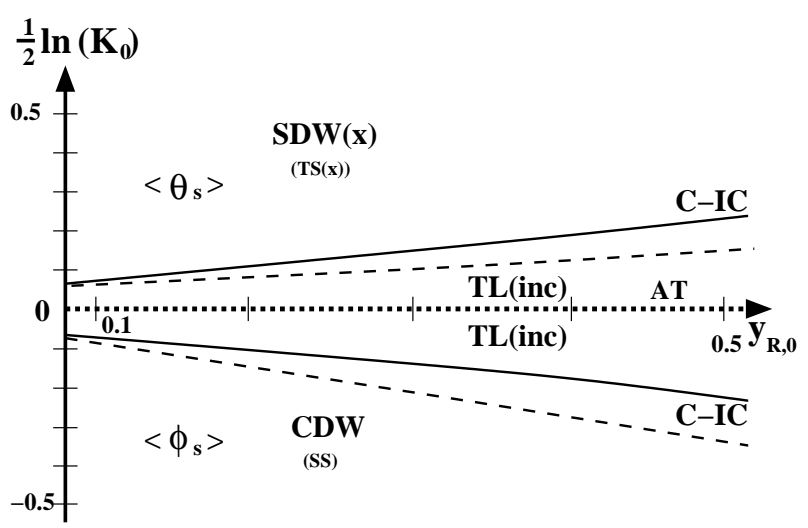

FIG. 2: Phase diagram in the parameter space of initial values $y_{R, 0}$ and $\frac{1}{2} \ln \left(K_{0}\right)$ for $y_{\perp, 0}=0$ (full lines), $y_{\perp, 0}=0.4$ (dashed lines), and for $y_{f, 0}=0$. The figure exhibits clearly the dual symmetry of Eq. (6). Spin gaps exist both in the phase with dominant superconducting correlations (SS or TS(x)) and in the spin (charge)-density wave phases $(\mathrm{SDW}(\mathrm{x})$ or $\mathrm{CDW})$. In the Luttinger-liquid phase (TL(inc)) all correlation functions decay algebraically. The TL phase is separated from the two spin-gapped phases by commensurate-incommensurate transitions (C-IC lines). The bold dotted line (AT at $K_{s}=1$ ) corresponds to the critical Ashkin-Teller model.

phase diagram in the parameter space $\left(y_{R, 0}, \frac{1}{2} \ln K_{0}\right)$ for the initial values $y_{\perp, 0}=0,0.4$ and $y_{f, 0}=0$. The straight line $\ln K_{0}=0$ (fixed points (II)) represents the self-dual line for $y_{\perp, 0}=y_{f, 0}=0$. The region of the incommensurate Luttinger-liquid phase widens as a function of $y_{R, 0}$. Outside of this region, the mean values $\left\langle\phi_{s}\right\rangle$ or $\left\langle\theta_{s}\right\rangle$ are finite, the former for $K_{0}<1$, the latter for $K_{0}>1$. In the $\phi_{s}$-ordered phase the dominant correlations are singlet superconductivity (SS, for $K_{c}>1$ ) and charge-density waves $\left(\mathrm{CDW}\right.$, for $\left.K_{c}<1\right)$. In the $\theta_{s}$-ordered phase the dominant correlations are the $x$-component of spindensity waves $\left(\mathrm{SDW}_{x}\right.$, for $\left.K_{c}<1\right)$ and the $x$-component of triplet superconductivity $\left(\mathrm{TS}_{x}\right.$, for $\left.K_{c}>1\right)$. The transition lines in Fig. 2 have been determined approximately on the basis of the RG flow. We note that the exact characterization of these commensurate-incommensurate transitions would require a non-perturbative analysis, which goes beyond the present perturbative RG scheme.

Spin precession is described by the correlation function 


$$
f(x)=\frac{1}{2}\left\langle\left(\psi_{\uparrow}(x)+\psi_{\downarrow}(x)\right)\left(\psi_{\uparrow}^{\dagger}(0)+\psi_{\downarrow}^{\dagger}(0)\right)\right\rangle .
$$

For a narrow, ballistic quantum wire connecting a source at $x=0$ to a drain at $x=L$ the quantity $|f(L)|^{2}$ measures the probability for a particle entering the drain to have the same spin orientation as one leaving the source. Within the Tomonaga-Luttinger model $f(x)$ is found [7] to vary as $|x|^{-\gamma}$, where $\gamma=(1 / 4)\left(K_{c}+1 / K_{c}+K_{s}+1 / K_{s}\right)$ depends on the electron-electron interaction through the charge and spin stiffnesses $K_{c}$ and $K_{s}$, respectively. The same behavior is expected to occur in our case within the Luttinger-liquid phase except that $K_{s}$ is replaced by the (non-universal) fixed-point value $K_{s}^{*}$. In principle, the presence of irrelevant couplings $y_{\perp}$ and $y_{f}$ can lead to extra multiplicative corrections [6] to $f(x)$, but they appear to vanish identically in our case. In the spingapped regime where the ordering of the $\phi_{s}$ field implies the disordering of $\theta_{s}$ field for $K_{s}<1$ (and vice versa for $K_{s}>1$ ), the function $f(x)$ is expected to decay as the corresponding correlator in the sine-Gordon model [16], namely $f(x) \sim x^{-(\lambda+1)} \exp \left(-x / \xi_{s}\right)$, where $\xi_{s}=\hbar v_{F} / \Delta_{s}$ is the correlation length, and $\lambda=\left(\sqrt{K_{c}}-1 / \sqrt{K_{c}}\right)^{2} / 4$.

We note that the spin stiffness $K_{s}$ is an important parameter in the problem. In $S U(2)$-invariant models with repulsive interactions $K_{s}$ scales to the fixed-point value $K_{s}^{*}=1$. This is no longer true for systems with spin-orbit coupling where the spin-rotation symmetry is reduced to $U(1)$.

As an example we consider a narrow InAs quantum wire with $m^{*}=0.023 m_{e}$ and $\alpha_{R}=(0.6-4) \times 10^{-11}$ $\mathrm{eVm}$ [1]. We choose the wire width $d=5 \mathrm{~nm}$ and the Fermi wave vector $k_{F}=0.5 \times 10^{8} \mathrm{~m}^{-1}$. In this case the assumption of a single occupied subband is justified. The parameter $y_{R, 0}$ depends on the cut-off length $\alpha$, for which a natural choice is the width $d$. Using these values and $u_{s} \approx v_{F}$ we find that $y_{R, 0}$ ranges from $10^{-4}$ to $10^{-2}$. In order to estimate $K_{0}$, we use the well-known relation [6] between the spin stiffness and the Fourier transform $V_{\text {eff }}(q)$ of the effective interaction potential for quantum wires. The latter is taken in the form proposed in Ref. 17]. Thus we obtain $\frac{1}{2} \ln K_{0} \approx 0.15$. According to Fig. 2 this corresponds to the spin-gapped phase. The standard procedure for the sine-Gordon model $[6]$ yields a value of $\Delta_{s}$ in the range $(0.01-0.1) \varepsilon_{F}$, where $\varepsilon_{F} \approx 4 \mathrm{meV}$ for given $m^{*}$ and $k_{F}$. For temperatures $T \leq \Delta_{s} / k_{B}=0.5-5$ $\mathrm{K}$ and wire lengths exceeding the correlation length, $L \geq$ $\xi_{s}=(0.4-4) \mu \mathrm{m}$ the phenomena described above will play an important role. These regions can be reached in present-day devices $(L \approx 2-6 \mu \mathrm{m})$, and it should in principle be possible to detect signatures of the spin gap.

If the material parameters can be tuned close to the commensurate-incommensurate transition from a Luttinger liquid to a phase with a spin gap, the dependence of the Rashba coupling on the electric field strength may allow to drive the system from one side of the transition to the other. Such a control of a spin gap by a gate voltage would represent a spectacular novel field effect. The observation of such a subtle phenomenon would of course not only be of fundamental interest, but it could also pave the way for the fabrication of new types of devices.

In conclusion, we have found that the interplay between electron-electron interactions and Rashba spinorbit coupling in a narrow wire generates a spin gap $\Delta_{s}$ for a certain range of parameters. This restricts the operation of the Datta-Das transistor. In the Luttingerliquid phase, where correlation functions fall off according to power laws, the device may work, but in the spingapped phase the spatial coherence of spin precession is suppressed exponentially, and the device efficiency tends to zero at lengths greater than the correlation length $\xi_{s}$.

We are grateful to Thierry Giamarchi and Gerd Schön for valuable discussions. V.G. was supported by the Swiss National Science Foundation through grant Nr.2068047.02. G.I.J. and D.B. acknowledge support through the SCOPES grant Nr. 7GEPJ62379. M.P. was supported by the DFG Center for Functional Nanostructures at the University of Karlsruhe.

[1] I. Žutić, J. Fabian, and S. Das Sarma, Rev. Mod. Phys. 76, 323 (2004); R. H. Silsbee, J. Phys.: Condens. Matter 16, R179 (2004).

[2] S. Datta and B. Das, Appl. Phys. Lett. 56, 665 (1990).

[3] Y.A. Bychkov, E.I. Rashba, J. Phys. C 17, 6039 (1984).

[4] J. Nitta, T. Akazaki, H. Takayanagi, and T. Enoki, Phys. Rev. Lett. 78, 1335 (1997); Y. Kato, R.C. Myers, A.C. Gossard, and D.D. Awschalom, Nature 427, 50 (2004).

[5] A.V. Moroz, K.V. Samokhin, and C.H.W. Barnes, Phys. Rev. Lett. 84, 4164 (2000); Phys. Rev. B 62, 16900 (2000); A. Iucci, Phys. Rev. B 68, 075107 (2003).

[6] T. Giamarchi, Quantum Physics in One Dimension (Oxford, 2004).

[7] W. Häusler, Phys. Rev. B 63, 121310(R) (2001).

[8] This can be checked within the perturbative scheme used in this paper.

[9] A.O. Gogolin, A.A. Nersesyan, and A.M. Tsvelik, Bosonization and Strongly Correlated Systems (Cambridge UP, 1998).

[10] S.A. Brazovskii and V.M. Yakovenko, J. Phys. Lett. (Paris) 69, 46 (1985); Sov. Phys. JETP 62, 1340 (1985); A.A. Nersesyan, A. Luther, and F. Kusmartsev, Phys. Lett. A 176, 363 (1993);

[11] T. Giamarchi and H.J. Schulz, J. Phys. France 49, 819 (1988); Phys. Rev. B 33, 2066 (1986).

[12] D. Boyanovsky, J. Phys. A: Math. Gen. 22, 2601 (1989).

[13] J.V. José, L.P. Kadanoff, S. Kirkpatrick, and D.R. Nelson, Phys. Rev. B 16, 1217 (1977).

[14] T.T. Truong and K.D. Schotte, Phys. Rev. Lett. 47, 285 (1981); Phys. Rev. B 24, R5426 (1981).

[15] G.I. Japaridze and A.A. Nersesyan, JETP Lett. 27, 334 (1978); V.L. Pokrovsky and A.L. Talapov, Phys. Rev. Lett. 42, 65 (1979).

[16] S. Lukyanov and A. Zamolodchikov, Nucl. Phys. B 607, 437 (2001).

[17] W. Häusler, L. Kecke, and A. H. MacDonald, Phys. Rev. B 65, 085104 (2002). 